\title{
The Star Cluster Systems of the Magellanic Clouds
}

\author{
G. S. Da Costa \\ Research School of Astronomy \& Astrophysics, ANU, Mt. Stromlo \\ Observatory, Cotter Rd, Weston ACT 2611, Australia
}

\begin{abstract}
The characteristics of the cluster systems of the Magellanic Clouds, as inferred from integrated properties, are compared with those from individual cluster studies and from the field population. The agreement is generally satisfactory though in the case of the LMC, the lack of clusters older than $\sim 3 \mathrm{Gyr}$ is not reflected in the field population. The possible origin(s) for this cluster "age-gap" are discussed. The SMC cluster age-metallicity relation is also presented and discussed.
\end{abstract}

\section{Introduction}

The star cluster systems of the Magellanic Clouds play a pivotal role in fulfilling this Symposium's aim: "to gain a comprehensive picture of star cluster formation and evolution, and their role in the evolutionary framework of their parent galaxies". This is because the relative proximity of the Magellanic Clouds means that LMC and SMC star clusters can be studied using both integrated light techniques and methods that rely on the light of individual cluster stars. We can then investigate the answer to the question "Do inferences made from integrated cluster properties agree with those from detailed star-by-star analyses?" Obviously the answer is important for studies of more distant cluster systems where only integrated light techniques are possible. Similarly, the proximity of the Magellanic Clouds makes it also possible to study the field star, as distinct from the cluster, populations of these galaxies. We can seek then to answer the question "Do results determined from the characteristics of the cluster system, such as 'Star' Formation Histories, agree with those for the general field population?". Again the answer is vital if the assumption that star cluster systems are surrogates for field populations is to produce valid results.

These questions underlie the basic approach of this contribution, in which we will compare the results of studies of the integrated properties of the Magellanic Cloud cluster systems, i.e., those properties an observer in a distant galaxy might determine, with the results from studies of individual LMC and SMC clusters, and of the field star populations. We begin with the LMC, and then discuss the SMC for which there are generally fewer data available.

\section{The Large Magellanic Cloud}

Figure 1 shows in the left panel, a colour-magnitude (c-m) diagram, and in the right panel, a $(U-B, B-V)$ 2-colour diagram, for LMC star clusters using 

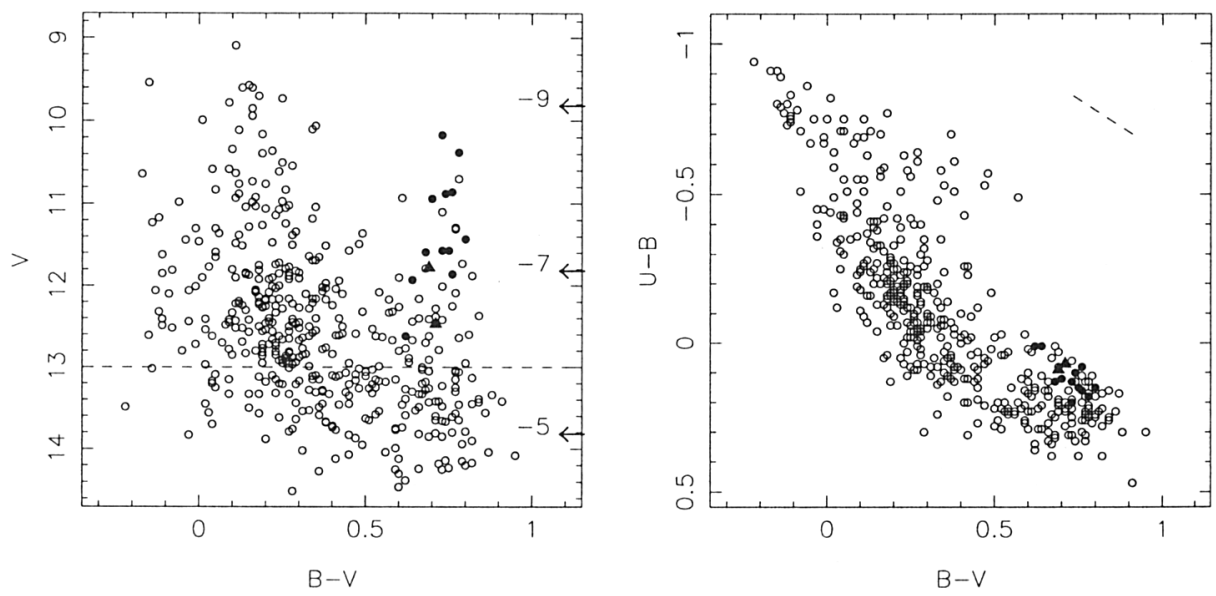

Figure 1. Integrated photometry for LMC Star Clusters from Bica et al. (1996). In both panels filled circles are confirmed old (age $>10$ Gyr) clusters while the filled triangles are the candidate old clusters from Dutra et al. (1999). Absolute magnitudes for $(m-M)_{0}=18.5$ and $E(B-V)=0.10 \mathrm{mag}$ are indicated on the right side of the left panel. The horizontal dashed line in the left panel is the approximate completeness level. The dashed line in the right panel shows the effect of a reddening change of $0.2 \mathrm{mag}$.

the data of Bica et al. (1996). Our distant observer of the LMC cluster system would immediately be able to conclude from these data that cluster formation has been on-going in the LMC, but to be more quantitative, it is necessary to compare the observations with single stellar population (SSP) models. In what follows we make use of the models (and results) of Girardi et al. (1995). These authors have calculated SSP models for a variety of abundances and find that they can achieve satisfactory agreement between the model tracks and the Bica et al. (1996) observational sample for abundances appropriate for the LMC.

\subsection{Clusters younger than $\sim 1 \mathrm{Gyr}$}

Based on the SSP models of Girardi et al. (1995), stars clusters younger than $\sim 1$ Gyr are those with $(B-V)_{0} \leq \sim 0.50 \mathrm{mag}$. The observed age distribution function for these clusters represents the global cluster formation history modified by the effects of observational selection (likely to be a minor issue here), fading as clusters age, and cluster disruption processes. The fading of clusters with age can be compensated for in a straightforward manner via SSP models, but allowance for the variety of potential cluster disruption processes is more difficult. Nevertheless, it is reasonable to assume that cluster disruption processes vary with age in a smooth way, and thus narrow features, such as peaks in the observed cluster age distribution at particular times, can be reasonably interpreted as relative increases in the true cluster formation rate at those times.

Girardi et al. (1995) determined the observed age distribution function as follows. As did Elson \& Fall (1985), they first drew a fiducial curve through 
the cluster points in the right panel of Fig. 1. An age dependent parameter $s$ is then defined as the distance along this fiducial line. The relation between $s$ and age is fixed by determining ages, using the same theoretical models as are incorporated in the SSP models, for a set of clusters with well determined c-m diagrams. The cluster points are then projected back to the fiducial line and a histogram distribution of $s$ values, or equivalently an age distribution, generated. Girardi et al. (1995) differ from earlier work (cf. Elson \& Fall) in that the projection vectors were derived by using the SSP models to calculate, as a function of cluster age, the effects on the integrated colours of stochastic variations in the numbers of cluster stars. These variations reproduce well the scatter seen in the left panel of Fig. 1.

The resulting observed cluster age distribution (cf. Figs. 12 and 15 of Girardi et al. 1995) has prominent peaks, which represent relative enhancements in the cluster formation rate, at ages of $\sim 10$ and $100 \mathrm{Myr}$. The location of these peaks is in remarkedly good agreement with the peaks in the observed cluster age distribution given by Pietrzyński \& Udalski (2000; these proceedings). These authors have individually age-dated approximately 600 LMC star clusters using c-m digrams derived from the OGLE photometry database. The peak in the relative number of clusters at ages around $\sim 100 \mathrm{Myr}$ also has a correspondance in the field star data. For example, Frogel \& Blanco (1983), among others, have used the colours and luminosities of LMC red giants in the LMC Bar to infer an era of enhanced (field) star formation at approximately this epoch.

Thus for this range of ages, the integrated cluster, the individual cluster, and the field star studies all give similar results.

\subsection{Clusters older than $\sim 1 \mathrm{Gyr}$}

2.2.1 The (very) old population. The LMC has 13 (possibly 15, see Dutra et al. 1999) genuine old globular clusters (e.g., Suntzeff et al. 1992). The integrated data for these clusters, i.e., their colours and spectra, are generally consistent with those for the Galactic halo globular clusters (e.g., Searle et al. 1980), supporting a strong degree of similarity. Indeed, based on c-m diagrams derived from HST/WFPC2 images, these LMC clusters appear to have the same age as Milky Way globular clusters to within a precision of approximately $\pm 1.5 \mathrm{Gyr}$ (Olsen et al. 1998; Johnson et al. 1999; Johnson et al., these proceedings).

However, as shown in Fig. 1, the LMC analogues of the Galactic halo globular clusters are not readily distinguished from the numerous intermediate-age $(\sim 1 \leq$ age $\leq \sim 10 \mathrm{Gyr})$ LMC star clusters in either the $(V, B-V)$ or the $(U-B, B-V)$ diagrams. Indeed it would be brave (or foolish) for our "distant observer" to attempt to identify, using these data alone, which of the LMC clusters with red $(B-V)$ integrated colours are the analogues of the Galactic halo globular clusters. Nevertheless, it is possible to make some progress in this direction by: (i) restricting the sample of clusters to the more luminous objects in order to reduce the effect of stochastic variations (i.e., sampling errors) on the integrated colours; (ii) determining individual reddenings for each cluster; and (iii) adding observations in near-IR wavebands. In star clusters younger than the Galactic halo globular clusters, the evolution of asymptotic giant branch stars continues to luminosities above that of the red giant branch tip. These upperAGB stars are cool and so have their greatest influence on near-IR colours. Fig. 2 

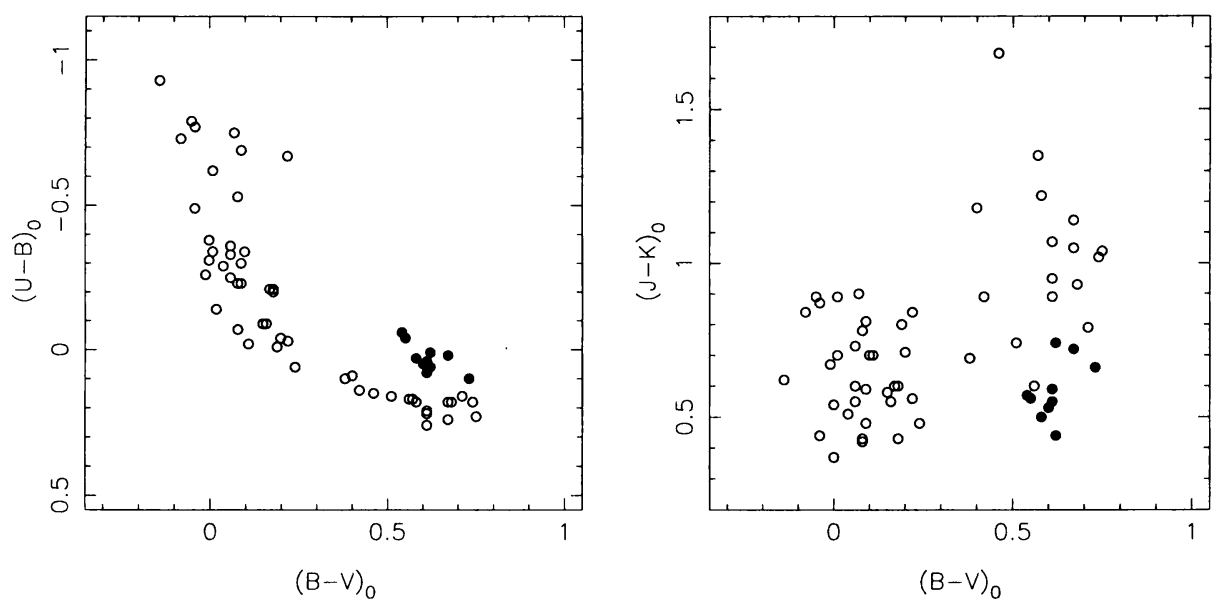

Figure 2. Integrated photometry for LMC Star Clusters from Persson et al. (1983). In both panels filled circles are confirmed old (age > $10 \mathrm{Gyr}$ ) clusters. It is clear that these diagrams allow the separation of old clusters from intermediate-age clusters.

shows $\left((U-B)_{0},(B-V)_{0}\right)$ and $\left((J-K)_{0},(B-V)_{0}\right)$ 2-colour diagrams for a restricted set of LMC star clusters using the data from Persson et al. (1983). In these diagrams the genuine old LMC star clusters can be separated from the intermediate-age clusters, as the latter have redder $(J-K)_{0}$ and $(U-B)_{0}$ for a given $(B-V)_{0}{ }^{1}$. As regards our distant observer then, these data allow the conclusion that there is a significant age range amongst the "red" LMC star clusters.

The field population of the LMC is known to contain RR Lyrae stars and thus it does contain a component which is old (age $\geq 10 \mathrm{Gyr}$ ). However, establishing the exact age of this old field population is by no means simple. Alcock et al. $(1996,2000)$, based principally on the properties of a large sample of LMC field RR Lyraes, have argued that the old horizontal branch in the LMC has a predominantly red morphology. For a mean metal abundance $\langle[\mathrm{Fe} / \mathrm{H}]\rangle \approx$ -1.6 dex, (Alcock et al. 1996) and assuming that age is the second parameter governing horizontal branch morphology, this would imply that the old metalpoor field population is 1-2 Gyr younger than the old metal-poor LMC cluster population (Alcock et al., 1996, 2000). Certainly numerous field c-m diagram studies have not revealed any significant numbers of blue horizontal branch stars in any part of the LMC, though such stars are found in the old globular clusters. Direct confirmation via field $\mathrm{c}-\mathrm{m}$ diagram studies of the assertion that the old field is younger than the old clusters in the LMC is a difficult process. Even in the outskirts of the LMC, the field population tends to be dominated by intermediate-age stars, so that separating out and dating the old population from the range of ages and metallicities present is far from straightforward.

\footnotetext{
${ }^{1}$ The clear separation of the old and intermediate-age clusters in Fig. 2 may well be due to the lack of clusters in the LMC with ages between $\sim 3$ and $10 \mathrm{Gyr}$.
} 


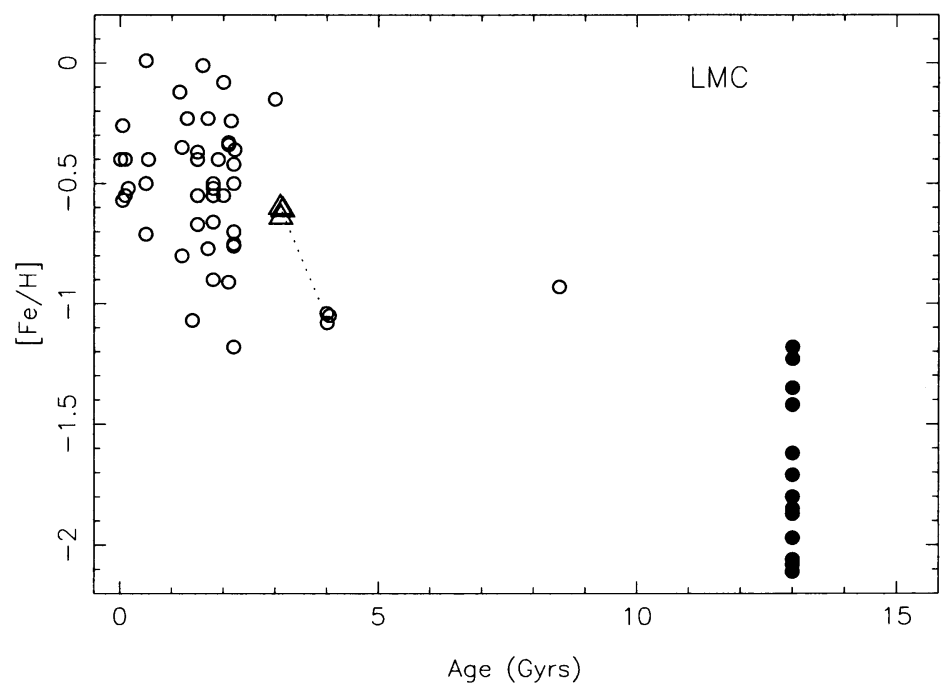

Figure 3. An Age-Metallicity relation for LMC star clusters. The 13 old LMC globular clusters have been assumed to be 13 Gyr in age. Open symbols represent data from Geisler et al. (1997), Bica et al. (1998) and Dirsch et al. (2000). The triangles represent the new results of Rich et al. (2001) for the clusters NGC 2121, SL663 and NGC 2155. The dotted line connects these values with the older results of Sarajedini (1998) for the same clusters. The isolated point at age 8.5 Gyr is the cluster ESO121-SC03.

However, using HST/WFPC2 based c-m diagrams, Walker et al. (1999) have found an age of $\sim 12 \mathrm{Gyr}$ for the old field stars in an outer LMC field near the cluster NGC 2257 . They see little need for any additional older ( $15 \mathrm{Gyr})$ component and thus their results tend to support the "old field is younger than the old clusters in the LMC" assertion.

In summary, it appears that for these (very) old ages, the integrated cluster results agree with those for the individual clusters and for the field, though the level of precision inherent in this statement is considerably less than for younger ages.

2.2.2 The intermediate-age population. The LMC cluster system is famous for its "age (and metallicity) gap". This is illustrated in Fig. 3 - the only cluster known to have an age between the age of the old globular clusters and $\sim 3 \mathrm{Gyr}$ is the sparse cluster ESO121-SC03. New VLT/UVES spectroscopy of giants in this cluster has confirmed its metal abundance as $[\mathrm{Fe} / \mathrm{H}] \approx-0.9$ dex (Hill et al. 2000). The Girardi et al. (1995) analysis of the $U B V$ integrated colours of the LMC clusters not only reveals a dearth of clusters in the LMC with ages in excess of $\sim 3 \mathrm{Gyr}$, but also suggests that the period between $\sim 700 \mathrm{Myr}$ and $\sim 3 \mathrm{Gyr}$ was an era with an enhanced cluster formation rate (cf. Figs. 12 \& 15 of Girardi et al. 1995). So once again the inferences from the integrated cluster data are consistent with those derived from individual cluster c-m diagram studies. 
2.2.3 The cluster "age-gap". The observed age distribution of LMC clusters with intermediate-age has undoubtedly been modified by the processes that lead to cluster disruption. However, cluster disruption does not seem to be a plausible complete explanation for the age-gap. There are two reasons for this. First, given that the amount of evolutionary fading between $\sim 3$ and 12 Gyr is relatively small ( $<1 \mathrm{mag}$ ), it is possible to infer from the left panel of Fig. 1 that at least some of the LMC intermediate-age clusters have comparable masses to the old globular clusters. They are therefore likely to survive for at least a few more Gyr, and hence, by inference, if clusters older than 3 Gyr had been formed in similar numbers they should have been discovered by now. Certainly clusters with ages exceeding $3 \mathrm{Gyr}$ are known in the SMC (found without any special search techniques), while targeted searches for age $>3$ Gyr clusters in the LMC have been notably unsuccessful (e.g., Geisler et al. 1997). Second, the number of 1-3 Gyr clusters in the LMC is comparatively large (at least 40 known) and the cutoff in numbers at $\sim 3$ Gyr is relatively sharp. It strains credulity to suggest the abrupt decrease in numbers at this particular age is solely a disruption effect. Hence, the inference from Fig. 3 is that the cluster formation rate was relatively low, perhaps even zero, for ages between $\sim 3$ Gyr and the formation epoch of the LMC Galactic halo globular cluster analogues. The question then is, what does the field population show - is the field star formation rate in the $\sim 3-10$ Gyr interval also relatively low (or zero) as the cluster data appear to imply?

There have been a number of studies recently which bear on this question. Those of particular relevance are based on HST/WFPC2 images which provide accurate data at faint magnitudes. Olsen (1999) has studied the star formation history in five fields (near old globular clusters) four of which lie in the Bar of the LMC and one in the LMC disk. Similarly, Holtzman et al. (1999) have studied two outer disk and one Bar field (see also Geha et al. 1998). The data for the Bar fields of both Olsen (1999) and Holtzman et al. (1999) show a marked (factor of $\sim 3$ ) increase in the star formation rate (SFR) at $\sim 5-6$ Gyr compared to earlier epochs, but with no particular evidence for any additional increase in the SFR rate at $\sim 3$ Gyr. Nevertheless, the SFR in these fields prior to $\sim 5-6$ Gyr is evidently not zero. The results for the disk fields are similar - there is no obvious increase in the SFR at $\sim 3$ Gyr and, while the SFR at epochs earlier than $\sim 5 \mathrm{Gyr}$ is evidently lower than for later epochs, it is clearly non-zero. Consequently, in contrast to what one might have been tempted to conclude from the existence of the cluster age-gap, the LMC field studies show that a significant fraction of the LMC field population is older than $\sim 3 \mathrm{Gyr}$.

Clearly then we have a case where inferences from the integrated and individual-star cluster studies give an incomplete picture of the star formation history of the underlying galaxy. This outcome should be kept in mind when analyzing the data for more distant systems: the properties of the cluster population may not be revealing the full history of the parent galaxy.

As regards the origin of the LMC cluster age gap, an explanation may lie in the work of Larsen \& Richtler (2000) and Larsen (these proceedings). Larsen \& Richtler (2000) show that the formation of massive star clusters is closely linked to star formation activity, and is favoured at higher star formation rates. The absolute magnitude of the brightest (young) massive star cluster also correlates with the star formation rate in the underlying galaxy (Larsen, these 
proceedings). Hence, in the LMC, it may be that the apparently lower overall star formation rate at ages beyond $5 \mathrm{Gyr}$ meant that not only were fewer clusters formed, but also that the clusters which were formed were less massive and thus more readily disrupted. Such a combination of effects could readily explain the existence of the cluster age-gap. Admittedly there is a difference between the onset of increased star formation in the LMC Bar at $\sim 5 \mathrm{Gyr}$, and the edge of the cluster-age gap at $\sim 3$ Gyr. The majority of the 1-3 Gyr old clusters studied, however, lie in the disk of the LMC rather than the Bar, and it may be that the epoch of increased star formation occurred later in the disk. For example, the Geha et al. (1998) study finds that the increase in the star formation rate in the outer disk of the LMC occured only about 2 Gyr ago.

\section{The Small Magellanic Cloud}

The cluster system of the SMC is less numerous than that of the LMC and, in general, has been less well studied. Nevertheless, there are some obvious differences between the SMC cluster system and that of the LMC. In particular, the SMC lacks any true "old, metal-poor" analogues to the globular clusters of the LMC and of the Galactic halo. The oldest SMC cluster (NGC 121) is $~ 2$ Gyr younger than the LMC and Galactic halo globular clusters (e.g., Shara et al. 1998). Further, with an abundance of $[\mathrm{Fe} / \mathrm{H}] \approx-1.45 \mathrm{dex}$, it is not notably metal-poor. Also in contrast to the LMC, the SMC cluster system shows no indication of any "age gap". Indeed, there are numerous SMC clusters with ages between 4 and $10 \mathrm{Gyr}$, which have no counterparts in the LMC.

Fig. 4 shows the age-metallicity relation for the SMC cluster system. This figure includes new data from Piatti et al. (2001; these proceedings) while the other cluster points are drawn from Da Costa \& Hatzidimitriou (1998), Mighell et al. (1998), de Freitas Pacheco et al. (1998), Rich et al. (2000) and earlier data. The "morphology" of this SMC cluster age-metallicity relation could be considered to have three components: (i) an initial (ages $\geq \sim 10 \mathrm{Gyr}$ ) enrichment up to $[\mathrm{Fe} / \mathrm{H}] \approx-1.5 \mathrm{dex}$; (ii) a long $(\sim 3 \leq$ age $\leq \sim 10 \mathrm{Gyr})$ interval where cluster age and abundance are not obviously correlated; and (iii) a relatively abrupt increase in the cluster abundances up to approximately the presentday abundance of the SMC field stars. This abundance increase seems to have commenced $\sim 2-3$ Gyr ago.

As discussed in Da Costa (1999), this SMC cluster age-metallicity relation cannot be readily understood in terms of either simple chemical models (cf. Da Costa \& Hatzidimitriou 1998), or by "bursting models" such as that of Pagel \& Tautvaisiene (1998). The latter models rely on a very low star formation rate in the 4-12 Gyr interval in order to reproduce the flat part of the age-metallicity relation. However, this assumed very low star formation rate is completely inconsistent with the existence of many rich star clusters that have formed during this epoch. The most likely explanation for the flat section of the relation is the infall of significant amounts of relatively unenriched gas. Further, Fig. 4 indicates that over much of the SMC's history there are real cluster-tocluster abundance differences. For example, the $\sim 0.3$ dex abundance difference between Kron 3 and NGC 339 is seen in both spectroscopic and photometric data. Dwarf galaxies like the SMC, provided the star formation rate is relatively 


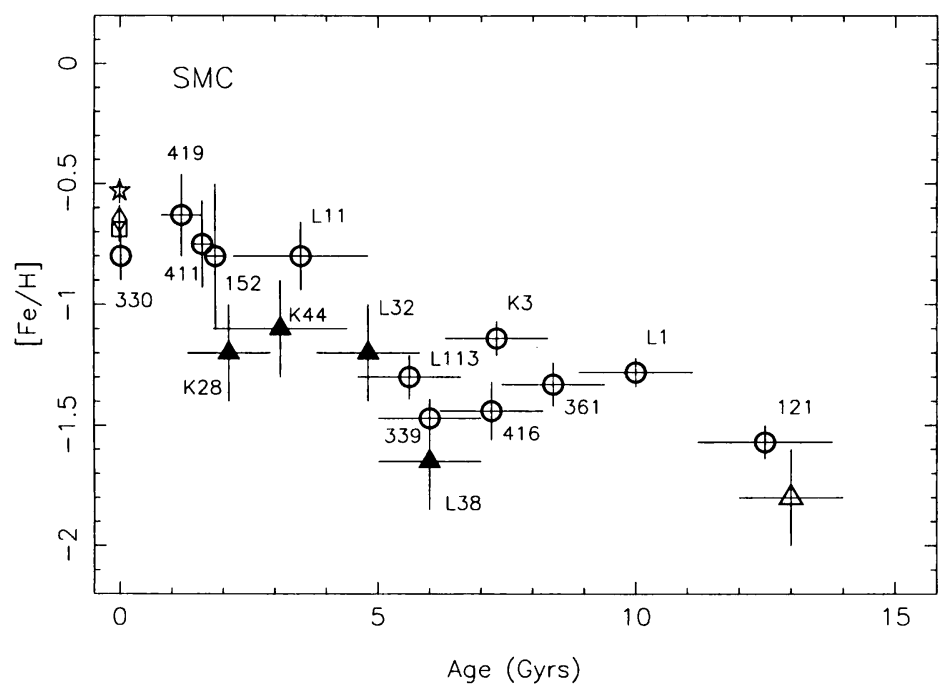

Figure 4. The Age-Metallicity relation for SMC star clusters. The four clusters studied by Piatti et al. (2001; these proceedings) are plotted as filled triangles; other clusters are plotted as open circles. Each cluster is identified by name. The open triangle represents SMC field RR Lyrae stars and the open diamond, square and star symbols are different estimates of the present-day field star abundance (see Da Costa \& Hatzidimitriou 1998 for details). Rich et al. (2000) have suggested on the basis of HST/WFPC2 snapshot images that NGC 152 and 411, and NGC 339, 416, Kron 3 and NGC 361 are in fact coeval.

constant and the infall of external material insignificant, are usually assumed to be chemically homogeneous, as the timescale to thoroughly mix the interstellar medium over dwarf-galaxy-wide scales is considerably shorter than a Hubble time (cf. Da Costa \& Hatzidimitriou 1998, Da Costa 1999). Apparently this is not the case in the SMC and the explanation may again lie with infall.

As regards the integrated properties of SMC clusters, the less numerous cluster population and the lack of a study comparable to that of Bica et al. (1996) mean that only general conclusions can be drawn. Based on the data of van den Bergh (1981), the equivalent diagrams for the SMC cluster system are broadly similar to those in Fig. 1. In particular, it is evident that cluster formation has been on-going and that a similar dichotomy into "blue" and "red" clusters at $(B-V) \approx 0.50$ exists. The data of Persson et al. (1983) show that the younger (i.e., age $\sim 1-3 \mathrm{Gyr}$ ) intermediate-age clusters NGC 152, 411 and 419 can be readily separated from the older (age $\geq \sim 6 \mathrm{Gyr}$ ) intermediate age-clusters such as NGC 339 and Kron 3 , by their red $(J-K)_{0}$ colours in the $\left((J-K)_{0},(B-V)_{0}\right)$ diagram (cf. Fig. 2). Thus it is possible to infer an extended age range among the SMC "red" clusters from their integrated properties. However, the $\left((J-K)_{0},(B-V)_{0}\right)$ diagram does not separate clusters like NGC 339 and Kron 3 from older clusters such as NGC 121, or from the LMC analogues of the Galactic halo globular clusters. The upper-AGB stars in the 
6-10 Gyr old clusters are simply not luminous enough to significantly redden the near-IR colours relative to those for older clusters. This result should be kept in mind when dealing with integrated cluster colours - it is often difficult to discern whether a particular cluster is $\sim 6-10$ Gyr old, or $\sim 12-15$ Gyr old (i.e., age comparable to the Galactic halo globular clusters) from integrated colours alone, even if near-IR colours are also available.

As regards the SMC field population, there is very little published data which reaches faint limiting magnitudes. Suntzeff et al. (1999) report that in a region near NGC 121, active star formation seems to have continued from an age approximately that of the Galactic halo globular clusters until about $6 \mathrm{Gyr}$ ago, when the star formation rate decreased substantially. Unfortunately, given the limited data, it is not really possible to assess the extent to which the field star formation history and the cluster formation history in the SMC agree, or disagree, though the clear inference from the discussion in Sect. 2.2.3 is that field star formation rate in the SMC should exceed that in the LMC in the $\sim 5-9$ Gyr interval. Much further work remains to be done in this area.

\section{Summary}

Clearly we are still some way from a complete understanding of the histories of the Magellanic Clouds, our nearest galactic neighbours. Neverthess, in the context of this meeting, it is possible to conclude that: first, the integrated properties of the cluster systems do give a reasonably accurate representation of the overall characteristics in comparison with those inferred from individual cluster studies. However, the inferences become less precise with increasing age, especially beyond a few Gyr. Second, the LMC cluster results suggest that one should be careful when interpreting cluster formation histories (even after allowing for disruption processes) in terms of overall star formation rates for the underlying galaxy. An absence of clusters (not related to disruptive processes) does not necessarily mean an absence of star formation.

\section{References}

Alcock, C., et al. 1996, AJ, 111, 1146

Alcock, C., et al. 2000, AJ, 119, 2194

Bica, E., Clariá, J. J., Dottori, H., Santos, J. F. C., Jr., Piatti, A. E. 1996, ApJS, 102, 57

Bica, E., Geisler, D., Dottori, H., Clariá, J. J., Piatti, A. E., Santos, J. F. C., Jr. 1998, AJ, 116, 723

Da Costa, G. S. 1999, in IAU Symp. 190, New Views of the Magellanic Clouds, ed. Y.-H. Chu, N. B. Suntzeff, J. E. Hesser \& D. A. Bohlender (San Francisco: ASP), 397

Da Costa, G. S., \& Hatzidimitriou, D. 1998, AJ, 115, 1934

de Freitas Pacheo, J. A., Barbuy, B., \& Idiart, T. 1998, A\&A, 332, 19

Dirsch, B., Richtler, T., Gieren, W. P., \& Hilker, M. 2000, A\&A, 360, 133

Dutra, C. M., Bica, E., Clariá, J. J., \& Piatti, A. E. 1999, MNRAS, 305, 373 
Elson, R. A. W., \& Fall, S. M. 1985, ApJ, 299, 211

Frogel, J. A., \& Blanco, V. M. 1983, ApJ, 274, L57

Geha, M. A., et al. 1998, AJ, 115, 1045

Geisler, D., Bica, E., Dottori, H., Clariá, J. J., Piatti, A. E., Santos, J. F. C., Jr. 1998, AJ, 114, 1920

Girardi, L., Chiosi, C., Bertelli, G., \& Bressan, A. 1995, A\&A, 298, 87

Hill, V., Francois, P., Spite, M., Primas, F., \& Spite, F. 2000, A\&A, 364, L19

Holtzman, J. A., et al. 1999, AJ, 118, 2262

Johnson, J. A., Bolte, M., Stetson, P. B., Hesser, J. E., \& Sommerville, R. S. 1999, ApJ, 527, 199

Larsen, S. S., \& Richtler, T. 2000, A\&A, 354, 836

Mighell, K. J., Sarajedini, A., \& French, R. S. 1998, AJ, 116, 2395

Olsen, K. A. G. 1999, AJ, 117, 2244

Olsen, K. A. G., Hodge, P. W., Mateo, M., Olszewski, E. W., Schommer, R. A., Suntzeff, N. B., \& Walker, A. R. 1998, MNRAS, 300, 665

Pagel, B. E. J., \& Tautvaisiene, G. 1998, MNRAS, 299, 535

Persson, S. E., Aaronson, M., Cohen, J. G., Frogel, J. A., \& Matthews, K. 1983, ApJ, 266, 105

Piatti, A. E., Santos, J. F. C., Jr., Clariá, J. J., Bica, E., Sarajedini, A., \& Geisler, D. 2001, MNRAS, 325, 792

Pietrzyński, G., \& Udalski, A. 2000, Acta Astronomica, 50, 337

Rich, R. M., Shara, M., Fall, S. M., \& Zurek, D. 2000, AJ, 119, 197

Rich, R. M., Shara, M. M., \& Zurek, D. 2001, AJ, 122, 842

Sarajedini, A. 1998, AJ, 116, 738

Searle, L., Wilkinson, A., \& Bagnuolo, W. G. 1980, ApJ, 239, 803

Shara, M. M., Fall, S. M., Rich, R. M., Zurek, D. 1998, ApJ, 508, 570

Suntzeff, N. B., Schommer, R. A., Olszewski, E. W., \& Walker, A. R. 1992, AJ, 104,1743

Suntzeff, N. B., et al. 1999, in IAU Symp. 190, New Views of the Magellanic Clouds, ed. Y.-H. Chu, N. B. Suntzeff, J. E. Hesser \& D. A. Bohlender (San Francisco: ASP), 397

van den Bergh, S. 1981, A\&AS, 46, 79

Walker, A. R., et al. 1999, in IAU Symp. 190, New Views of the Magellanic Clouds, ed. Y.-H. Chu, N. B. Suntzeff, J. E. Hesser \& D. A. Bohlender (San Francisco: ASP), 341

\section{Discussion}

R. Kennicutt: What are the current results on the age distribution in the LMC Bar, and its association (if any) with the onset of cluster formation 3 Gyr ago?

G. Da Costa: If you combine the data for Olsen's four "Bar" fields, then the average star formation rate in the 1-3 Gyr interval is approximately a factor of 
two larger than it was over the 3-9 Gyr interval. It is also a factor of $\sim 4$ larger than the average SFR in the 5-9 Gyr interval. So the Bar is dominated by stars younger than about 5 Gyr. The Holtzmann et al. (1999) data suggest similar results. The increased SFR in the Bar seems to have commenced somewhat earlier than the increase in the rate of massive cluster formation, though I suspect this difference may be related to the fact that most of the 1-3 Gyr old clusters are in the LMC disk not the Bar.

J. Frogel: 1 . Is there a difference in $[\mathrm{Fe} / \mathrm{H}]$ distribution of LMC and Milky Way RR Lyrae that could account for the inferred age difference? 2. If you look at specific cluster frequency per number of field stars, is the "age-gap" still really a gap?

G. Da Costa: 1. Alcock et al. (1996) give an $[\mathrm{Fe} / \mathrm{H}]$ distribution based on spectra of only 15 LMC field RR Lyraes. This distribution, which peaks at $[\mathrm{Fe} / \mathrm{H}] \approx$ -1.6 with a prominent tail to lower abundances and an abrupt cutoff at $[\mathrm{Fe} / \mathrm{H}]$ $\approx-1.0$, is broadly similar to the $[\mathrm{Fe} / \mathrm{H}]$ distribution for Galactic halo field $\mathrm{RR}$ Lyrae. But a larger sample of LMC field RR Lyrae abundances is needed to give statistical weight to this similarity (or any difference). 2 . If the specific frequency of clusters is constant, then since the overall (Bar and disk) star formation rate in the 1-3 Gyr interval is $\sim 3$ times that in the 3-9 Gyr interval, we would expect roughly equal numbers of 1-3 Gyr and 3-9 Gyr old clusters if we ignore fading and disruption. Fading ( $\leq 1 \mathrm{mag}$ from 3 to $9 \mathrm{Gyr}$ ) will reduce the relative number of older clusters in any magnitude limited (i.e., observed) sample and undoubtedly a number of (potential) older clusters, particulary those of lower mass, will have been disrupted. Yet we know of at least 40 clusters whose ages, determined from main sequence turnoff photometry, lie in the 1-3 Gyr range while, despite significant searches (e.g., Geisler et al. 1997), there is only one LMC cluster known whose age exceeds $3 \mathrm{Gyr}$ and which is younger than the LMC Galactic globular cluster analogues (ESO 121-SC03). It seems to me highly unlikely that this disparity results solely from a combination of fading and disruption. The "age-gap" is a real phenomenon.

H. Lamers: Is it possible that the presence of an age gap for the LMC clusters, and the lack of an age gap for the field stars, is due to the fact that most clusters with age $>3$ Gyrs have dispersed? In my (poster) paper I show that the disruption time depends on the initial cluster mass as $t_{d i s} \sim \mathrm{M}^{+0.6}$, so the lower mass clusters may have dispersed and only the most massive ones survived.

G. Da Costa: Disruption of LMC clusters must play some role in generating the "age-gap". However, I do not believe that disruption processes can be entirely responsible for the relatively abrupt cutoff in the cluster age distribution at $\sim 3$ Gyr. There have been a number of extensive searches for LMC clusters with ages in excess of $\sim 3 \mathrm{Gyr}$ and all have been singularly unsuccessful. Further, a number of current 1-3 Gyr old LMC clusters have luminosities that indicate initial masses exceeding $10^{4} \mathrm{M}_{\odot}$ and thus, using the results from your poster, disruption times in excess of $5 \mathrm{Gyr}$. It is then difficult to see, if LMC clusters had formed at a uniform rate, why the age gap would exist. 